\title{
Space Time Coding Techniques in MIMO: A Review
}

\author{
Shakti Raj Chopra*, Navjot Kaur and Himanshu Monga \\ Department of Electronics and Communication Engineering, Lovely Professional University \\ Phagwara-144411,Punjab, India; shakti.chopra@lpu.co.in, navjot.12695@|pu.co.in, \\ himanshumonga@gmail.com
}

\begin{abstract}
This paper explore the basic approaches used for of space-time coding technique. The emerging field of wireless evaluation such as Multiple Input Multiple Output (MIMO) and Antenna diversity plays an important role in space-time code design. Spatial diversity techniques by the means of multiple transmitting and receiving antennas and the performance of MIMO systems has been discussed for different space-time coding structures such as Alamouti, OSTBC3, OSTBC4 etc. Space-time coding techniques rely on the construction of matrices and the signal is to be transmitted at different period of time from multiple of antennas. These code structures utilises the diversity schemes to improve the bit error rate and thus to achieve higher data rates without bandwidth expansion.
\end{abstract}

Keywords: BER, Multiple Input Multiple Output (MIMO), OSTBC3, OSTBC4, SNR, Spatial Diversity, STBC, STTC

\section{Introduction}

In MIMO technology improves the performance of system through multiplexing and diversity, achieved high data rates and antenna as compared to single antenna. The system uses multiple antenna at input (transmitter) side and the same as at output (receiver) side is commonly referred as MIMO systems. Capacity of the wireless systems is improved using MIMO also link additional bandwidth and power requirements.

The wireless communication when compared to wire line communication leads to two fundamental aspects as mentioned below:

- In wired communication, point to pointcommunication between every transmitter-receiver pair is considered whereas in wireless communication, communication is possible through the air.

- Fading phenomenon that occurs in the wireless communication as the channel strength changes with time due multipath fading and path loss via distance attenuation and by obstacles shadowing.

\subsection{MIMO}

In MIMO system an arrangement of various antennas at both sender and receiver ends. In MIMO system antennas are separated spatially has a basic privilege of the spatial in a dense multipath environment which involves scattering. A MIMO system is used in various ways to obtain diversity gain to overcome fading in the signal to have a high gain in capacity. Normally, MIMO system is categorized into different techniques. The main aim to maximizing spatial diversity so that power efficiency can be improved. These types of can be approach embraces delay diversity, STBC $\underline{12}$ and STTC $\underline{\underline{3}}$, another approach named as layered approach has been used to increase capacity V-BLAST ${ }^{4}$ is the example of this approach.

\subsection{Space-Time Block Coding Techniques}

STBC is the basic codes through which offered diversity in systems at the transmitter side with several transmit antennas.

\subsubsection{Alamouti Space Time Coding}

In 1998, Alamouti ${ }^{1}$ gave the designing of a simple diversity technique at the transmission end systems

${ }^{*}$ Author for correspondence 
having only two transmit antennas. The requirement of this method is that it needs linear operations both at the transmitting and receiving ends and hence provides full diversity. The methods to encode and decode the data were performed by using blocks of various transmission symbols.

Let the two modulated symbols be denoted by $\mathrm{x}_{1}$ and $\mathrm{x}_{2}$ which are passed to the space-time encoder. Commonly systems having only single antenna at the transmitting side, let symbols $\mathrm{x}_{1}$ and $\mathrm{x}_{2}$ are transmitted at different time $t_{1}$ and $t_{2}$ consecutively. Here, in this Alamouti scheme, for time instance $t_{1}$, both antenna element transmits the symbol $x_{1}$ and $\mathrm{x}_{2}$, respectively. At $\mathrm{t}_{2}$ time instant, the first antenna transmit the conjugate of first ie $x_{1}^{\square}$. and second antenna transmits the negative conjugate of symbol in i.e., $-\mathrm{x}_{2}^{\square}$.

The following coding matrix represents Alamouti's transmit diversity technique for two branch:

$$
\mathrm{X}_{1}=\left[\begin{array}{cc}
\mathrm{x}_{1} & -\mathrm{x}_{2}^{*} \\
\mathrm{x}_{2} & \mathrm{x}_{1}^{*}
\end{array}\right]
$$

The transmit antennas are represented by the coding matrix rows while the different time instances are represented by coding matrix columns.

\subsubsection{Orthogonal Space Time Coding}

Alamouti gave the designing of one of the types of space diversity scheme that is transmit diversity where the number of transmitting antennas is confined to two this scheme results STBC or, Orthogonal STBCs to be more precise, as they also include the principle of orthogonality ${ }^{5}$. V. Tarokh et $\mathrm{al}^{\underline{6}}$ which created codes for number of antennas at the transmitting end using this scheme.

It reflected the orthgonality property of STBCs because all the rows are orthogonal to one another ${ }^{\text {? }}$. Orthogonal signal is which are perpendicular to each other will not affect and transmitted using distinct antenna . It is achievable to have full rate for real signal. But this scheme is not correct for complex signals as proved by ${ }^{6}$. The approaches to encode and decode the data follows the schematic mentioned in the Alamouti's scheme. The theory of orthogonal designs generates coding matrices for complex signals which has a rate of transmission as $1 / 2$ in the cases where between 3 and 4 antennas were used at the transmitting end.

$$
\mathrm{x}_{\frac{1}{2}}=\left[\begin{array}{cccccccc}
\mathrm{x}_{1} & -\mathrm{x}_{2} & -\mathrm{x}_{3} & -\mathrm{x}_{4} & \mathrm{x}_{1}^{*} & -\mathrm{x}_{2}^{*} & -\mathrm{x}_{3}^{*} & -\mathrm{x}_{4}^{*} \\
\mathrm{x}_{2} & \mathrm{x}_{1} & \mathrm{x}_{4} & -\mathrm{x}_{3} & \mathrm{x}_{2}^{*} & \mathrm{x}_{1}^{*} & \mathrm{x}_{4}^{*} & -\mathrm{x}_{3}^{*} \\
\mathrm{x}_{3} & -\mathrm{x}_{4} & \mathrm{x}_{1} & \mathrm{x}_{2} & \mathrm{x}_{3}^{*} & -\mathrm{x}_{4}^{*} & \mathrm{x}_{1}^{*} & \mathrm{x}_{2}^{*}
\end{array}\right]
$$

$$
\mathrm{x}_{\frac{1}{2}}=\left[\begin{array}{cccccccc}
\mathrm{x}_{1} & -\mathrm{x}_{2} & -\mathrm{x}_{3} & -\mathrm{x}_{4} & \mathrm{x}_{1}^{*} & -\mathrm{x}_{2}^{*} & -\mathrm{x}_{3}^{*} & -\mathrm{x}_{4}^{*} \\
\mathrm{x}_{2} & \mathrm{x}_{1} & \mathrm{x}_{4} & -\mathrm{x}_{3} & \mathrm{x}_{2}^{*} & \mathrm{x}_{1}^{*} & \mathrm{x}_{4}^{*} & -\mathrm{x}_{3}^{*} \\
\mathrm{x}_{3} & -\mathrm{x}_{4} & \mathrm{x}_{1} & \mathrm{x}_{2} & \mathrm{x}_{3}^{*} & -\mathrm{x}_{4}^{*} & \mathrm{x}_{1}^{*} & \mathrm{x}_{2}^{*} \\
\mathrm{x}_{4} & \mathrm{x}_{3} & -\mathrm{x}_{2} & \mathrm{x}_{1} & \mathrm{x}_{4}^{*} & \mathrm{x}_{3}^{*} & -\mathrm{x}_{2}^{*} & \mathrm{x}_{1}^{*}
\end{array}\right]
$$

\subsubsection{Quasi Orthogonal Space Time Coding}

By employing transmit beam forming scheme in which at the transmitting end channel information is available, the analysis of a multiple-antenna system can be made better. A new performance criterion was proposed by Jongren et al on the basis of partial channel side information at the transmitter. Using the given approach, for orthogonal space-time block codes, the optimal beam forming matrix was constructed. However, because of the nonorthogonal nature of the quasi-orthogonal designs ${ }^{56}$ the technique has not rejected for the (QSTBCs)

H.Jafarkhani et $\mathrm{al}^{9}$ pointed out the issue of the combination of beam forming with QSTBCs. On the basis of asymptotic analysis; the beam forming technique was extended from Jongren et al.,the beam forming matrices was constructed for the quasi-orthogonal scheme. Due to application of this proposed beam forming scheme the increased rate of transmission and spatial diversity was achieved. The new QSTBC beam former which works for systems comprising of transmit antennas equal to or more than four has been proposed as four-directional or eightdirectional eigen-beam former. A notable improvement in performance over various other widely used beam forming methods at different Signal to noise ratios(SNR's) and channels having different feedback quality can be seen with the simulations for systems having various antennas at the transmitting side.

\subsubsection{Space-Time Trellis Codes}

Space- time trellis codes are used in wireless communications where multiple antennas are to be used. Under this approach various copies of convolution or trellis code are scattered and rely over time from different spatially placed antennas. The reassemble of actual rely data is perform at the receiving end with the aid of the various, different data copies. The need of multiple transmit antennas is mandatory for an STC to be used. In this scheme only one antenna needed however various receive antennas can be implemented due to which the system performance will increase.

STBCs provides both coding and diversity gain and it has an increased value of BER. Whereas on the basis of trellis code, it appears to be more complex than 
STBCs to encode and decode and at the receiver side it needs a Viterbi decoder while STBCs need only linear processing.

An effective alternative signaling technique is provided by STTCs. By embedding the trellis or convolution code being embedded into the design of STTC for better coding gain, and diversity on Rayleigh fading channels as well as spectral efficiency can be obtained simultaneously.

\section{Literature Review}

S. Alamouti ${ }^{1}$ presented the diversity technique having number of transmitting antennas are two and have only one receive antenna and examine the diversity order and find the same as two receive antennas, and one transmit antenna using maximal-rati combining with one transmit particular scheme can be derived for two transmit antennas and $\mathrm{M}$ receive antennas to provide a diversity order of $2 \mathrm{M}$ without the expansion of bandwidth .

V. Tarok $\frac{6,15}{}$, design a channel codes using various relay antennas for enhance the reliability and the throughput of communications over fading channels. Assuming that the fading is slow and frequency non-selective the authors derived the performance criteria for designing such codes. The Trellis code has been designed for the high data rate wireless communication particular design criteria are used.

H. Jafarkhani ${ }^{9}$ compared the quasi-orthogonal space-time block code (QO-STBC with an orthogonal space-time block code such as Alamouti code. Quasi orthogonal codes areprovide full transmission rate. The quasi orthogonal codes are those in which different groups are orthogonal to each other although each groups are not and the transmission metric column divided into groups. As compared to full diversity orthogonal code quasi orthogonal codes have better performance (lower BER) at low SNR's whereas their performance is degrading at higher SNR's.

N. Sharma et.al $\underline{10}^{10}$ discussed about significant improvement in the performance of Quasi-orthogonal ST codes is achieved if we phase shift or rotate the constellations of the codes symbols. The basis of constellation rotation is that it increases the minimum distance between the ST code words thus improving performance. Particularly at low BER's performance gain of $6 \mathrm{db}$ and 4 $\mathrm{db}$ have been obtained for QPSK \& 8PSK respectively as compared to no rotation.
The QOSTBC are designed properly to obtain better performance even at increased values of Signal to Noise Ratio in which resulting codes perform the orthogonal codes in items of diversity decoding and SNR as given by W. Su et.al ${ }^{11}$.

The concept of transmit beam forming is considered \& a beam forming matrix is constituted for STBC. The concept of beam forming is extended by L Liu et.all ${ }^{12}$ for QSTBC by which has not been implemented yet. The resulting QSTBC beam forming has high rate and degree of spatial diversity.

In the paper given by $\mathrm{H}$. Jafarkhani et.al ${ }^{13}$ a family of QOSTBC are considered as building block for Quality of Service (QOS) ST Trellis codes. The QOSTBC are designed by amalgamate set portioning and a super set of QOSTBC and provide full rate diversity and maximize coding gain.

The scheme of existing complex orthogonal STBC for 7 transmitting antennas is extended for 8transmitting antennas by X.B. Liang $\frac{14}{}$ which achieves same rate and decoding delay.

The STTC $\frac{15}{2}$ gives the performance of wireless MIMO better to much extent by using multiple transmitter antennas both at the receiver as well as at the receiver. The rank and the determinate of the codeword distance matrix, at present, are the critical parameters in the slow fading channels. The rank is used to find the diversity gain, whereas the determinant criterion is used for achieving code gain. There are a number of space time codes with the improved performance that has been proposed. Space time trellis code was proposed byBaro, et al ${ }^{16}$ analyzed $\mathrm{BBH}$ codes, the analysis of which is better than Tarokh et al (TSC) $)^{15}$ codes. Chen et al [17] CYV codes which ruled by the lowest trace matrices and the minimum Euclidean distance among two codeword overall transmit antenna. CYV codes has been shown to give better analysis than $\mathrm{TSC}^{\frac{15}{5}}$ and $\mathrm{BBH}^{\frac{16}{16}}$ codes.

Further improvement in error of the space time codes are a subject of research. Mainly the focus is on the MIMO system with beam forming, antenna selection and antenna grouping for the improvement of the error performance. Space time code selection $\frac{18}{18}$ is a scheme that adjusts the space time code to the number of antennas. It allows the transmitter to adapt to CSI by using small feedback information that is achieved when switching takes place for the space time code selected for the number of transmitter antennas limited up to four. It is proved that the scheme for the selection of the code 
provides a gain in the performance with trade off of the complexity. The space time trellis codes $\frac{19}{}$ are designed by combination of a standard M-TCM outer code with inner code. Channel phase feedback has been used for selecting inner code from series inner codes. The criteria for selection of inner code are based on the decoding of low peak to average power ratio and can be easily implemented. $\stackrel{20}{ }$ Balanced STBC implemented using a small number of bits at the sender side that is on the basis of the information of the channels. approach gives improvement in SNR compared to the Alamouti's approach with antenna selection.

An adaptive code selection scheme ${ }^{21}$ is presented that increases the signal to noise ratio at the destination. In this scheme it is shown that adaptive code selection using four bit feedback improves signal to noise ratio at the destination by the use of extended balanced space time block and at the transmitter end it has proper channel state information.

Antenna selection is another technique $e^{22,23}$ that gives result of high potential diversity order using simple encoding and decoding with lower complexity. This technique can be applied both at the receiver and at the sender side. A simple scheme for selection of the antenna is Receive Antenna Selection for an non coded SingleInput Multiple-Output system, in which only one receiver antenna with the highest SNR.

Transmitter selection of antenn $\mathrm{a}^{24,25}$ is another scheme for in this a part of transmit antennas is used for sending. The selection of transmit antenna is generally uses space time codes made for a less number of antennas. This scheme of selecting transmitter antenna has been explored with help of STBC and STTC. In antenna transmitting the convolution code with space time code such as TAS/STBC ${ }^{24,25}$ and TAS/STTC ${ }^{26}$ for antenna selection, the antenna should be modified such that I became a globally optimized antenna such that it became best choice when compared among all the available antennas without any restriction. Considering this TAS/STBC/STTC feature recommended this antenna to have better code availability and error performance.

Partitioning of transmitting antennas into small subgroups, space-time codes has been used individual transmitter transmitted information from each subgroup of transmit antennas. For decoding linear array processing technique has been used for decode so that it suppresses signal that is transmitted from any other group of antennas as it treat all other signals as interference.
The selected group of transmitting antenna is in accordance with the channel state feedback. This grouping of the antennas is checked at the receiving side and at the transmitting side the index of the result is fed back to it in the form of a codebook. This grouping of adaptive antenna improves the SNR. Beamforming block code has been proposed in $27-28$. All such techniques have attained improved system performance by beam forming gain as well as coding gain. The space time trellis codes and beam forming codes has been combined in ${ }^{29-30}$ to design WSTTCs (weighted space time trellis codes). The results has shown this technique has achieved additional time diversity with full spatial diversity as well as additional receiver gain of signal to noise ratio and a better coding gain relative to STTS.

The bandwidth efficiency over AWGN channel is increased by the introduction of multilevel coding which uses several error correction codes. In this coding technique a combination of simple codes creates a complicated code. This technique $\mathrm{e}^{\underline{31}}$ uses both modulation and error correction coding to choose transmitting constellation points by using the component codes. The decoding of multilevel codes are done sequentially in various stages ${ }^{33}$ by allowing the decoded information to pass through from one stage to the next stage.

\section{Conclusion}

MIMO system can be implemented using M-PSK or M-QAM modulation techniques for high throughput, due to space diversity as the number of transmitting and receiving antennas increases, bit Error Rate (BER) decreases with different values of Signal-to-Noise Ratio (SNR) but needed much energy, to get a reliable wireless link. The spectral efficiency has been improved as increases Tx and Rx antenna.. Thus in order to increase the throughput and reliability of a communication system, digital modulation can be employed in multi antenna system, further improvement of bit error rate using trellis code to achieve full diversity and coding gain.

\section{References}

1. Alamouti S. A simple transmit diversity technique for wireless communications. IEEE Journal on Selected Areas of Communication. 1998; 16(8): 1451-58.

2. Toker C, Lambotharan S, Chambers JA. Closed-Loop QuasiOrthogonal STBCs and their performance in multipath 
fading environments and when combined with Turbo Codes. IEEE Transaction on Wireless Communications. 2014; 3(6): 1890-96.

3. Wu X, Jia-nian C, Rui Y. Design and analysis of low complexity Quasi Orthogonal Space-Time Block Code. IEEE Conference on Industrial Electronics and Applications. Xi'an, China: 2009. p. 3848-52. 20.

4. Wolniansky PW, Foschini GJ, Golden GD,Valenzuela RA. V-Blast: An architecture for realizing very high data rates over the rich-scattering channel. International Symposium on Signals, Systems and Electronics. 1998. p. 295-300.

5. Bien PV, Sheng W, Ma X,Wang H. Improved decoder schemes for QOSTBCs based on single-symbol decoding. International Conference on Advanced Technologies for Communications. Ho Chi Minh City: 2010. p. 7-10.

6. Tarokh V, Jafarkhani H,Calderbank AR. Space-time block codes from orthogonal designs. IEEE Transactions on Information Theory. 1999; 45(5): 1456-67.

7. Ganesan G, Stoica P. Space-Time block codes: a maximum SNR approach. IEEE Transactions on Information Theory. 2001; 47(4):1650-56

8. Tarokh V, Seshadri N,Calderbank AR. Space-Time codes for high data rate wireless communication: Performance criterion and code construction. IEEE Transactions on Information Theory. 1998; 44( 2): 744-65.

9. Jafarkhani HA Quasi-Orthogonal Space-Time Block Code. IEEE Transaction on Communications. 2001; 49( 1): 1-4.

10. Sharma N,Papadias CB. Improved Quasi-Orthogonal codes through constellation rotation. IEEE Transaction on Communications. 2003; 51(3): 332-5.

11. Su W,Xia XG. Signal constellations for Quasi-Orthogonal Space-Time Block Codes with full diversity. IEEE Transactions on Information Theory. 2004; 50(10): 2331-47.

12. Liu L, Jafarkhani H. Application of Quasi-Orthogonal space-time block codes in beamforming. IEEE Transactions on Signal Processing.2005; 53(1): 54-63.

13. Jafarkhani H,Hassanpour N. Super-Quasi-Orthogonal Space-Time Trellis Codes for Four Transmit Antennas. IEEE Transaction on Wireless Communications. 2005; 4(1): 215-27.

14. Liang XB. A Complex Orthogonal Space-Time block code for 8 transmit antennas. IEEE Communication Letters. 2005; 9(2): 115-7.

15. Tarokh V, Seshadri N, Calderbank AR. Space-Time Codes for high data rate wireless communication: performance criterion and code construction. IEEE Transactions on Information Theory. 1998; 44(2): 744-765.

16. Baro S, Bauch G, Hansmanna A. Improved codes for spacetime trellis-coded modulation. IEEE Communications Letters. 2000; 4: 20-22.

17. Chen Z, Vucetic B Yuan J. Improved Space-Time trellis coded modulation scheme on slow Rayleigh fading channels.Electronics Letters. 2001; 37: 440-1.
18. Mavares D, Torres RP. Space-time code selection for transmit antenna diversity systems. Proceedings of the First Mobile Computing and Wireless Communication International Conference. 2006; p. 83-7.

19. Liu L, Jafarkhani H.Space-time trellis codes based on channel-phasefeedback. IEEE Transactions on Communications. 2006; 54: 2186-98.

20. Celebi ME, Sahin S Aygolu U. Full rate full diversity spacetime block code selection for more than two transmit antennas.IEEE Transactions on Wireless Communications. 2007; 6: 16-19.

21. Eksim A,Celebi ME. Received SNR based code and antenna selection for limited feedback communication. Proceedings of 18th conference of IEEE Signal Processing and Communications Applications. 2010.p. 21-4.

22. Molisch AF, Win MZ. MIMO systems with antenna selection. IEEE Microwave Magazine. 2004; 5(1): 46-56.

23. Gore DA,Paulraj AJ. MIMO antenna subset selection with space-time coding. IEEE Transactions on Signal Processing. 2002; 50(10): 2580-88.

24. Wong WH,Larsson EG. Orthogonal space-time block coding with antenna selection and power allocation. Electronics Letters. 2003; 39(4): 379.

25. Tao M, Li Q,Garg HK. Extended Space-Time block coding with transmit antenna selection over correlated fading channels. IEEE Transactions on Wireless Communications. 2007; 6(9): 3137-41.

26. Chen Z, Vucetic B,Yuan J. Space-Time trellis codes with transmit antenna selection. Electronics Letters. 2003; 39(11): 854-5

27. Jongren G, Skoglund M,Ottersten B. Combining beam forming and orthogonal space-time block coding. IEEE Transactions on Information Theory. 2002;48: 611-27.

28. Zhou S,Giannakis G. Optimal transmitter eigen-beamforming and space-time block coding based on channel mean feedback. IEEE Transactions on Signal Processing. 2002; 50: 2599-613.

29. Li Y, Vucetic B, Santoso A, Chen Z. Space time trellis codes with adaptive weighting. Electronics Letters. 2003; 39: 1833-34. DOI: 10.1049/el:20031180

30. Santoso A, Li Y, Vucetic B. Weighted space time trellis codes. Electronics Letters. 2004; 40: 254-56.

31. Imai $\mathrm{H}$ and Hirakawa $\mathrm{S}$. A new multilevel coding method using error correcting codes. IEEE Transactions on Information Theory. 1977; 23(3): 371-7.

32. Calderbank A. Multilevel codes and multistage decoding. IEEE Transactions on Communications. 1989; 37(3): 222-9.

33. Waschmann U, Fischer RF,Huber JB. Multilevel codes: theoretical concepts and practical design rules. IEEE Transactions on Information Theory. 1999; 45(5): 1361-91. 